\title{
Eyelid Function Disorder, CTCAE
}

National Cancer Institute

\section{Source}

National Cancer Institute. Eyelid Function Disorder, CT CAE. NCI Thesaurus. Code C143471.

A disorder characterized by impaired eyelid function. 